Sitientibus Série Ciências Físicas 05: 1-5 (2009)

\title{
Álgebra de Lie das Matrizes Gama da Equação de Dirac e dos Geradores do Grupo de Lorentz Homogêneo
}

\section{Lie Algebra for the Gamma Matrixes from the Dirac Equation and for the Generators of the Homogeneous Lorentz Group}

\author{
Ricardo Martinho Lima Santiago Pereira* \\ Instituto de Física - UFBA \\ Campus Universitário de Ondina, $s / n$, \\ Salvador - BA - 40210-340
}

\begin{abstract}
Este artigo aborda a construção de uma álgebra de Lie a partir do estudo da equação quântico relativística de Dirac tomando a condição de invariância relativística frente ao grupo de Lorentz Homogêneo no espaço de Minkowiski. Com base no estudo da teoria dos grupos contínuos, demonstramos que o estabelecimento de uma álgebra de Lie é uma condição suficiente para determinar as representações irredutíveis de um grupo contínuo. A partir da exigência de covariância da equação de Dirac frente ao grupo de Lorentz Homogêneo podemos estabelecer uma álgebra para as matrizes gama induzida pela álgebra dos geradores do grupo de Lorentz Homogêneo.
\end{abstract}

Palavras-chaves: Álgebra de Lie, Equação de Dirac, Grupo de Lorentz Homogêneo.

This article does approach the construction of a Lie algebra from the study of Dirac's relativistic quantum equation taking into account the relativistic invariance condition of the homogeneous Lorentz group in the Minkowski space. It based on the study of the theory of continuous groups, we demonstrate that the establishment of a Lie algebra is a suffice condition to find the irreducible representations of a continuous group. From the requirement of the covariance for Dirac equation with respect to the homogeneous Lorentz group, we determine the algebra of the gamma matrixes induced by the algebra of the generators of homogeneous Lorentz group.

Key-words: Lie Algebra, Dirac Equation, Homogeneous Lorentz Group.

\section{INTRODUÇÃO}

Este trabalho pretende determinar uma álgebra de Lie a partir dos geradores infinitesimais do grupo de Lorentz Homogêneo junto com matrizes gama que figuram na equação quântica relativística de Dirac, funcionando estas como geradores infinitesimais do grupo contínuo assim determinado. Para tanto faremos algumas considerações sobre os grupos de Lie e, em particular, sobre o grupo de Lorentz Homogêneo e, em seguida, colocamos uma breve discussão acerca das equações quânticas relativísticas, incluindo a equação de Dirac.

*Endereço Eletrônico: vacuidade@gmail.com
Por fim passamos a determinação da álgebra de Lie definida pelas matrizes gama e os geradores infinitesimais do grupo de Lorentz Homogêneo.

Esses resultados foram conseguidos de forma pioneira por Ettore Majorana, para uma representação das matrizes gama usando as representações do Grupo de Lorentz Homogêneo, tendo sido um dos primeiros trabalhos que se valeu de uma aplicação do Grupo de Lorentz de Dimensão Infinita. Com isto, E. Majorana consegue seu intento de determinar um espectro de energia para partículas livres com autovalores exclusivamente positivos, dispensando-se assim da explicação de Dirac do mar de partículas confinadas em estados indetectáveis, o que eventualmente dava 
origem a noção de antipartículas. Como isto, a equação de Dirac pode ser escrita para valores arbitrários de spins inteiros e semi-inteiros.

\section{GRUPOS DE LIE (GRUPO DE LORENTZ HOMOGÊNEO)}

Como definido em Bassalo [1], "um grupo cujos elementos são caracterizados por um número de parâmetros contínuos é chamado Grupo Contínuo." (Bassalo, pg. 1). Entre os grupos contínuos nos é interessante definir os grupos de Lie assim:

"um grupo de r-parâmetros (r finito) é dito um grupo de Lie se:

$$
\begin{aligned}
& c_{\lambda}=\phi_{\lambda}\left(a_{1}, a_{2}, \ldots, a_{r} ; b_{1}, b_{2}, \ldots, b_{r}\right), \\
& \text { ou c } c=\phi(a ; b) \text { é uma função } \\
& \text { analítica, isto é, pode ser de- } \\
& \text { senvolvida em série de Taylor, } \\
& \text { uniformemente convergente, dos } \\
& \text { parâmetros a e b" ([1], pg. 74). }
\end{aligned}
$$

Para a Física, os grupos de simetria são muito importantes. Eles em geral representam a invariância dos sistemas frente a um conjunto de transformações, de um referencial inercial para outro. No caso clássico, são representadas pelo grupo de Galilei (translações espaciais e temporais, rotações e transformações de Galileu). No caso relativístico são representadas pelo grupo de Lorentz (translações e rotações no espaço de Minkowiski). Faremos agora um breve estudo do Grupo das transformações de rotação espaço-espaço e espaçotempo que constituem o Grupo de Lorentz homogêneo, assim como da álgebra dos geradores deste. Por este meio podemos definir que o conjunto de todas as transformações de rotação geométricas espaciais, junto com as transformações de referencias inerciais no formato proposto por Lorentz constituem o grupo de Lorentz Homogêneo, ou o grupo das Rotações Quadridimensional, tendo em vista que as transformações de referencial de Lorentz representam geometricamente rotações espaçotemporais.
Considerando que $R^{(3)}(\theta)$ é uma rotação de ângulo $\theta$ em torno do eixo $z$, definimos o gerador infinitesimal das rotações, representado por $a_{3}$, da seguinte forma:

$$
a_{3} \equiv\left(\frac{d R^{3}(\theta)}{d \theta}\right)_{\theta=0}
$$

De forma que uma rotação infinitesimal pode se escrita assim:

$$
R_{3}(\theta)=I+a_{3} \theta+\mathcal{O}\left(\theta^{n}\right)
$$

onde $n \geq 2$. Naturalmente para rotações finitas realizamos uma sucessão de rotações infinitesimais dadas por " $n$ " passos, com $n \rightarrow \infty$,

$$
R_{3}(\theta)=\lim _{n \rightarrow \infty}\left(I+\frac{\theta}{n} a_{3}\right)^{n}=\mathrm{e}^{a_{3} \theta} .
$$

Começamos pelo grupo das rotações espaciais cujos geradores, e suas representações, satisfazem a seguinte álgebra:

$$
\left[a_{j}, a_{k}\right]=-\epsilon_{j k m} a_{m}
$$

Sabemos que

"os geradores infinitesimais $\left\{X_{l}\right\}$ de qualquer Grupo de Lie, satisfazem às relações:

$$
\left[X_{\alpha}, X_{\beta}\right]=C_{\alpha \beta}^{\gamma} X_{\gamma},
$$

onde $C_{\alpha \beta}^{\gamma}$ são as chamadas Constantes de Estrutura do Grupo de Lie." ([1], pg. 82), e "um Grupo de Lie dotado da operação de comutação entre seus geradores infinitesimais é chamado de Álgebra de Lie, operação essa que satisfaz às seguintes propriedades:

a) $\left[X_{\alpha}, X_{\beta}\right]=-\left[X_{\beta}, X_{\alpha}\right]=C_{\alpha \beta}^{\gamma} X_{\gamma}$;

b) $\left[\left(\lambda X_{\alpha}\right), X_{\beta}\right]=\lambda\left[X_{\alpha}, X_{\beta}\right], \lambda \in \mathbb{R}$;

c) $\left[X_{\alpha},\left(X_{\beta}+X_{\gamma}\right)\right]=\left[X_{\alpha}, X_{\beta}\right]+\left[X_{\alpha}, X_{\gamma}\right]$;

d) $[(A+i B), C]=[A, C]+i[B, C]$,

onde $A, B, C$ são do tipo $a_{\rho} X_{\rho}$." ([1], pg. 92).

Contudo, como os geradores não comutam en- 
tre si, as definições a seguir permitem determinar os comutadores dos novos elementos,

$$
\begin{aligned}
A_{j} & \equiv i a_{j}, \quad \epsilon, \lambda \equiv \pm 1, \\
A_{\lambda} & \equiv A_{1}+i \lambda A_{2}, \\
A^{2} & \equiv \frac{1}{2}\left(A_{+} A_{-}+A_{-} A_{+}\right)+A_{3}^{2},
\end{aligned}
$$

assim obtemos,

$$
\begin{aligned}
& {\left[A_{j}, A_{k}\right]=i \epsilon_{j k m} A_{m},} \\
& {\left[A_{j}, A^{2}\right]=0,} \\
& {\left[A_{\lambda}, A_{3}\right]=-\lambda A_{\lambda},} \\
& {\left[A_{\lambda}, A_{-\lambda}\right]=-2 \lambda A_{3} .}
\end{aligned}
$$

Desta forma, estabelecemos operadores hermitianos cujos autovalores são sempre reais, uma condição necessária para corresponderem a um observável físico. Também verificamos, inicialmente, que os operadores $A^{2}$ e $A_{3}$ comutam, embora nenhum dos operadores $A_{j}$ comutem entre si, logo podemos estabelecer uma base comum usando os autovetores de $A^{2}$ e $A_{3}$. Por outro lado, tendo em conta (5),

$$
\left\langle k, m\left|A_{3} A_{\lambda}\right| k, m\right\rangle=(m+\lambda)\left\langle k, m\left|A_{\lambda}\right| k, m\right\rangle .
$$

Assim, os operadores $A_{1}$ e $A_{2}$ podem ser substituídos por $A_{+1}$ e $A_{-1}$, que tem os mesmos autovetores que $A_{3}$. Em outras palavras, os autovetores de $A^{2}$ e $A_{3}$ são suficientes para caracterizar o grupo das rotações espaço-espaço, o que completa o estudo dos geradores de rotações espaciais.

Reunimos, então, a estas as rotações espaçotemporais dadas pela transformação de Lorentz no Espaço de Minkowiski. Completando o grupo de Lorentz próprio cuja álgebra associada é [9]

$$
\begin{aligned}
& {\left[A_{j}, A_{k}\right]=i \epsilon_{j k m} A_{m},} \\
& {\left[B_{j}, B_{k}\right]=-i \epsilon_{j k m} A_{m},} \\
& {\left[A_{j}, B_{k}\right]=i \epsilon_{j k m} B_{m} .}
\end{aligned}
$$

Em analogia ao procedimento anterior, podemos estabelecer as seguintes definições,

$$
\begin{aligned}
& B_{j} \equiv i b_{j}, \quad \epsilon, \lambda \equiv \pm 1, \\
& B_{\lambda} \equiv B_{1}+i \lambda B_{2},
\end{aligned}
$$

que nos levam, após alguns cálculos, aos resultados para os comutadores abaixo,

$$
\begin{array}{lr}
{\left[B_{j}, A^{2}\right]=0,} & {\left[A_{3}, B_{3}\right]=0,} \\
{\left[A_{\lambda}, B_{\lambda}\right]=0,} & {\left[B_{\lambda}, A_{3}\right]=\lambda B_{\lambda},} \\
{\left[B_{\lambda}, B_{-\lambda}\right]=-2 \lambda A_{3}} & {\left[A_{\lambda}, B_{3}\right]=\lambda B_{\lambda},} \\
{\left[B_{\lambda}, A_{-\lambda}\right]=-2 \lambda B_{3}} & {\left[B_{\lambda}, B_{3}\right]=\lambda A_{\lambda} .}
\end{array}
$$

O que demonstra, com os três primeiros comutadores, que os operadores $B_{i}$ têm os mesmos autovetores que os operadores $A_{i}$, e que a partir destes podemos determinar todas as representações irredutíveis do grupo de Lorentz homogêneo.

\section{EQUAÇÕES QUÂNTICO-RELATIVÍSTICAS (EQUAÇÃO DE DIRAC)}

Conforme encontramos ao longo dos textos de J. Leite Lopes [7], Schweber [8], Naimark [9], Bjorken [10], a primeira equação proposta por Schroedinger para uma onda de matéria ou radiação escalar (a uma componente), foi feita levando em conta a relação de dispersão da energia de uma partícula livre em momento e massa da relatividade restrita,

$$
E^{2}=p^{2} c^{2}+m^{2} c^{4}
$$

embora, esta equação leve a uma segunda derivada temporal o que implica em valores positivos e negativos para a energia. Esta primeira equação hoje é conhecida como Equação de Klein-Gordon como segue

$$
\partial^{\mu} \partial_{\mu} \psi-\left(\frac{m c}{\hbar}\right)^{2} \psi=0 .
$$

Contudo, Schroedinger percebeu que poderia se livrar do incômodo das energias negativas construindo uma equação de onda escalar com base na expressão da energia não-relativística total de um sistema,

$$
i \hbar \frac{\partial \psi}{\partial t}=-\frac{\hbar^{2}}{2 m} \nabla^{2} \psi+U \psi
$$

que é a equação que leva seu nome.

Seguindo um caminho semelhante, Dirac 
propôs uma equação de onda espinorial de primeira ordem que satisfizesse a relação de dispersão para cada componente, e esta é a Equação de Dirac,

$$
\gamma^{\mu} \partial_{\mu} \psi-\left(\frac{i m c}{\hbar}\right) \psi=0
$$

Esta nova equação possui algumas propriedades interessantes e é bastante reveladora quanto ao Spin da partícula. Como estamos interessados, neste trabalho, na condição de invariância relativística perante o grupo de Lorentz Homogêneo ao aplicar uma transformação de Lorentz a esta equação obtemos

$$
\begin{aligned}
& \gamma^{\mu} \Lambda_{\mu}^{\nu} \partial_{\nu} D(\Lambda)^{-1} \psi-\left(\frac{i m c}{\hbar}\right) D(\Lambda)^{-1} \psi=0 \\
& D(\Lambda) \gamma^{\mu} \eta_{\alpha}^{\nu} \Lambda_{\mu}^{\alpha} \partial_{\nu} D(\Lambda)^{-1} \psi-\left(\frac{i m c}{\hbar}\right) \psi=0 \\
& D(\Lambda) \gamma^{\mu} \eta_{\alpha}^{\nu} \Lambda_{\mu}^{\alpha} \partial_{\nu} D(\Lambda)^{-1}=\gamma^{\nu}
\end{aligned}
$$

onde definimos as transformações de Lorentz e as representações das transformações de Lorentz $D(\Lambda)$ por

$$
\begin{aligned}
& x^{\nu} \equiv \Lambda_{\mu}^{\nu} x_{\mu}=\left(\delta_{\mu}^{\nu}+\epsilon \eta_{\mu}^{\nu}\right) x^{\mu}, \\
& D(\Lambda) \equiv\left(I+i \frac{\epsilon}{2} \lambda^{\alpha \mu}\right) M^{\alpha \mu}, \\
& A_{i} \equiv \frac{1}{2} \epsilon_{i j k} M_{j k}, \\
& B_{i} \equiv-i \epsilon_{i j k} M_{i 4} .
\end{aligned}
$$

Em função destas definições e da necessidade de invariância de equação de Dirac por qualquer transformação do Grupo de Lorentz Homogêneo, passamos agora a determinar a álgebra de Lie das matrizes gama e os geradores infinitesimais do grupo de Lorentz Homogêneo.

\section{IV. ÁLGEBRA DE LIE DAS MATRIZES GAMA E GERADORES DO GRUPO DE LORENTZ HOMOGÊNEO}

O que pretendemos é determinar o conjunto de comutadores dos geradores do grupo de Lorentz e as matrizes gama da equação de Dirac quântico relativística, e reunir aos comutadores envolvendo os geradores do grupo de
Lorentz, que consistem na álgebra de Lie deste grupo. Tomando a condição de invariância da equação de Dirac (11), observamos que esta pode ser reescrita cmomo segue

$$
\begin{aligned}
& \left(I+i \frac{\epsilon}{2} \lambda^{\alpha \mu}\right) M_{\alpha \mu} \gamma^{\mu} \eta_{\alpha}^{\nu} \Lambda_{\mu}^{\alpha}\left(I-i \frac{\epsilon}{2} \lambda^{\alpha \mu}\right)=\gamma^{\nu}, \\
& {\left[M_{\alpha \mu}, \gamma_{\nu}\right]=i\left(\eta_{\nu \alpha} \gamma_{\mu}+\eta_{\nu \mu} \gamma_{\alpha}\right) .}
\end{aligned}
$$

Então, temos para os geradores hermitianos $A_{i}$ e $B_{j}$,

$$
\begin{aligned}
& {\left[A_{i}, \gamma_{\nu}\right]=\frac{i}{2} \epsilon_{i j k}\left(\eta_{\nu j} \gamma_{k}+\eta_{\nu k} \gamma_{j}\right)} \\
& {\left[A_{i}, \gamma_{j}\right]=i \epsilon_{i j k} \gamma_{k}} \\
& {\left[A_{i}, \gamma_{4}\right]=0} \\
& {\left[B_{i}, \gamma_{\nu}\right]=\left(\eta_{\nu i} \gamma_{4}+\eta_{\nu 4} \gamma_{i}\right)} \\
& {\left[B_{i}, \gamma_{\nu}\right]=\delta_{\nu i} \gamma_{4}} \\
& {\left[B_{i}, \gamma_{4}\right]=-\gamma_{i}}
\end{aligned}
$$

O conjunto dos comutadores que constituem a álgebra de Lie deste grupo é

$$
\begin{array}{lr}
{\left[A_{i}, A_{k}\right]=\epsilon_{j k m} A_{m},} & {\left[B_{j}, B_{k}\right]=-i \epsilon_{j k m} A_{m},} \\
{\left[A_{j}, B_{k}\right]=\epsilon_{j k m} B_{m},} & {\left[A_{i}, \gamma_{j}\right]=i \epsilon_{i j k} \gamma_{k},} \\
{\left[A_{i}, \gamma_{4}\right]=0,} & {\left[B_{i}, \gamma_{\nu}\right]=\delta_{\nu i} \gamma_{4},} \\
{\left[B_{i}, \gamma_{4}\right]=-\gamma_{i} .} &
\end{array}
$$

\section{CONSIDERAÇÕES FINAIS}

Destacando os aspectos mais interessantes do trabalho, utilizamo-nos do fato de que a invariância relativística da equação de Dirac quântico relativística frente ao grupo de Lorentz Homogêneo estabelece uma álgebra de Lie junto às matrizes gama desta mesma equação.

Como as matrizes gama não têm uma representação única podemos determinar representações para estas a partir desta álgebra Lie que determinamos neste artigo, que é exatamente o que é feito na representação de Majorana.

A representação de Majorana determina uma matriz com autovalores todos positivos para a energia de partículas livres, e por conseqüência valores de massa exclusivamente positivos, requerendo para isso apenas a linearidade da equação de Dirac. Com isso se encon- 
tra um espectro de massa em função do spin arbitrário inteiro e semi-inteiro.

Em particular ele consegue o resultado ainda mais fantástico de determinar um espectro de massa das partículas elementares em função do spin, cuja variação condiz com os resultados experimentais, embora não os valores exatos. Neste sentido é bem sugestivo o estabelecimento de uma relação de dispersão de energia da massa em spin, tal como a relação de dispersão da energia em massa e momento linear.

Com a representação de Majorana podemos perceber a grande relevância para uma melhor compreensão da relação entre a massa e spin das partículas elementares, assim como a possibilidade de estabelecer uma descrição unificada entre bósons e férmions.

Este trabalho pode ser estendido com a aplicação ao estudo de propriedades estatísticas, ou, por outro viés, ao estudo dos pro- cessos de criação e aniquilação de partículas, aplicando a equação de Dirac na representação de Majorana, no estudo da quantização de um oscilador harmônico entre outras coisas.

O trabalho de Majorana tem destacado muita atenção na atualidade, tendo inclusive sua obra completa sendo republicada, a exemplo dos artigos de Espósito [15] e Fradkin [16] utilizados para este trabalho.

O estudo das representações lineares do grupo de Lorentz foi amplamente estudado no trabalho de Naimark [8] em continuidade ao estudo pioneiro de Majorana. E esta releitura pretende também servir como um chamamento aos estudantes para o interesse na área de pesquisa em apreço, para aqueles que pretendem se aprofundar no assunto.
[1] J.M.F. Bassalo, M.S.D. Cattani, Teoria de Grupos. São Paulo: Editora Livraria da Física (2008).

[2] T. S. Kuhn, A Estrutura das Revoluções Científicas. São Paulo: Editora Perspectiva S.A. (1997).

[3] L. Wittgenstein, Tractatus LogicoPhilosophicus. São Paulo: Editora da Universidade de São Paulo (1994).

[4] L. Landau, E.M. Lifshitz, Teoria do Campo. Moscou: Editora Mir (1980).

[5] H.M. Nussenzveig, Curso de Física Básica 1 Mecânica. São Paulo: Editora Edgard Blücher (1981).

[6] H.M. Nussenzveig, Curso de Física Básica 4 - Ótica, Relatividade e Quântica. São Paulo: Editora Edgard Blücher (1981).

[7] J. Leite-Lopes, A Estrutura Quântica da Matéria - do Átomo Pré-Socrático às Partículas Elementares, $2^{a}$ ed.. Rio de Janeiro: UFRJ Editora (1992).

[8] S. Schweber, An Introduction to Relativistic Quantum Field Theory. New York: Harper \& Row Publishers (1962).

[9] M.A. Naimark, Linear Representations of the
Lorentz Group. New York: The Macmillan Company (1964).

[10] J.D. Bjorken, S.D. Drell, Relativistic Quantum Mechanics. New York; McGraw-Hill (1964).

[11] J.W. Leech, Mecânica Analítica. Rio de Janeiro: Editora Guanabara - Ao Livro Técnico S.A. (1971).

[12] N.A. Lemos, Mecânica Analítica. São Paulo: Editora Livraria da Física (2004).

[13] J.B. Neto, Mecânica Newtoniana, Lagrangeana e Hamiltoniana. São Paulo: Editora Livraria da Física (2004).

[14] C. Cohen-Tannoudji, B. Diu, F. Lalöe, Quantum Mechanics. New York: Wiley-Interscience Publication (1977).

[15] R. Eisberg, R. Resnick, Relativistic Quantum Mechanics Rio de Janeiro: Elsevier (1979).

[16] S. Esposito, Four Variations on Theoretical Physics by Etorre Majorana. Eletronic Journal of Theoretical Physics 3, (10) 305 (2006).

[17] R.M.L.S. Pereira, A Equação de Dirac e A Representação de Majorana. In: Seminário de Iniciação Científica da UEFS, 2008. Feira de Santana: UEFS (2008). 